\title{
NITROGÊNIO E POTÁSSIO VIA FERTIRRIGAÇÃO E ADUBAÇÃO CONVENCIONAL-ESTADO NUTRICIONAL DAS BANANEIRAS E PRODUÇÃO DE FRUTOS ${ }^{1}$
}

\author{
LUIZANTONIO JUNQUEIRA TEIXEIRA² ${ }^{2}$ WILLIAM NATALE ${ }^{3}$, ANTONIO LÚCIO MELLO MARTINS $^{4}$
}

RESUMO - Realizou-se um experimento em Pindorama (SP) com o objetivo de avaliar os efeitos da fertirrigação e da adubação convencional com N e K, em bananeiras, durante dois ciclos de produção. Foram avaliados crescimento, estado nutricional e produção de frutos. A adubação causou redução do ciclo de produção. Os teores foliares de $\mathrm{N}$ e K foram influenciados pela adubação convencional e pela fertirrigação. Nos dois ciclos de cultivo, a produção de frutos variou em função dos tratamentos. A produção de frutos $\left(\mathrm{t}\right.$ ha ${ }^{1}$ ano ${ }^{1}$ ) obtida com a aplicação de $80 \%$ da dose de $\mathrm{N}$ e de $\mathrm{K}$ via fertirrigação foi equivalente àquela com $100 \%$ da dose via adubação convencional.

Termos para indexação: banana, análise foliar, cloreto de potássio, nitrato de amônio, Musa spp.

\section{NITROGEN AND POTASSIUM APPLICATION ON BANANA PLANT BY FERTIRRIGATION AND CONVENTIONAL FERTILIZATION-NUTRITIONAL STATUS OF BANANA PLANTS AND FRUIT PRODUCTION}

\begin{abstract}
A field experiment was carried out in Pindorama (Sao Paulo State, Brazil) with the objective of investigating the effects of $\mathrm{N}$ and $\mathrm{K}$ application through fertirrigation and conventional fertilization on banana plants during two crop cycles. Plant growth, nutrition status and fruit production were evaluated. The fertilization caused a reduction in the productive cycle. Fertilizers applied by fertirrigation or conventional fertilization changed the $\mathrm{N}$ and $\mathrm{K}$ leaf content. The fruit production varied in function of the treatment in the two cultivated cycles. Fruit production $\left(\mathrm{tha}^{1} \mathrm{year}^{1}\right)$ obtained with application of $80 \%$ of $\mathrm{N}$ and $\mathrm{K}$ doses by fertirrigation was comparable to fruit production with $100 \%$ of fertilizer rate applied by conventional fertlization.
\end{abstract}

Index terms: banana, foliar analysis, potassium chloride, Ammoniun nitrate, Musa spp.

\section{INTRODUÇÃO}

O Estado de São Paulo é o maior produtor nacional de bananas (IBGE, 2006); em 2005, de acordo com IEA (2006), foram colhidas cerca de 1,1 milhão de toneladas de frutos/ano, cultivadas em 54 mil ha, dos quais aproximadamente $65 \%$ se encontram no Vale do Ribeira. Destaca-se, também, a recente expansão da bananicultura para o Planalto Paulista. Áreas com cultivos tradicionais (café, pastagem, etc.) têm na fruticultura alternativa de alta rentabilidade e que traz importantes benefícios sociais, como o aumento da disponibilidade de alimentos de qualidade e a oferta de empregos. Entretanto, a sazonalidade das chuvas no Planalto Paulista torna a irrigação imprescindível para o cultivo de bananeiras nesta região. Segundo Lahav (1995), irrigação é um fator importante quando se estuda a nutrição de bananeiras. Se houver limitação no suprimento de água, a absorção de nutrientes, especialmente de $\mathrm{N}$, será reduzida. A despeito de sua importância, Lahav \& Turner (1983) e Lahav (1995) afirmaram que estudos relacionando irrigação e adubação em bananeira são escassos em nível mundial. Segundo Bar-Yosef (1999), irrigação e fertilização seriam os fatores passíveis de manejo mais importantes para o controle do desenvolvimento das plantas, do rendimento e da qualidade de frutos.
Fertirrigação é a prática de aplicar fertilizantes dissolvidos na água de irrigação de forma contínua ou intermitente. Além da disposição dos adubos na região de maior concentração de raízes e da possibilidade de maior fracionamento das doses, a fertirrigação possibilita aumentar a eficiência das adubações, pois os nutrientes têm as condições ideais de umidade do solo para sua absorção. Entretanto, a fertirrigação não se adapta a todos os sistemas de irrigação, visto que um dos pré-requisitos é operar com alta uniformidade de aplicação. Por isso, associa-se principalmente aos sistemas de irrigação por gotejo ou microaspersão (Yagüe, 1996). Para bananeiras, a irrigação por microaspersão é adequada às necessidades da cultura, pois, segundo Soto (1992), apresenta algumas vantagens, como a redução no molhamento de folhas, flores e frutos, pequena influência de ventos e trabalha com baixa pressão.

Hernandez (1994) afirmou que a fertirrigação no Brasil era utilizada de forma incipiente comparada ao seu potencial, destacando algumas vantagens desse sistema, como economia de mão-de-obra e energia, diminuição da compactação do solo, eficiência do uso e economia de fertilizante, controle da profundidade de aplicação, entre outras. Recentemente, Villas Bôas et al. (2005) consideraram que houve aumento da fertirrigação no Brasil e no mundo, pois a técnica se mostrou

\footnotetext{
1 (Trabalho 162-2006). Recebido em 19-10-2006. Aceito para publicação em 13-02-2007. Trabalho realizado com o apoio da FAPESP (Projeto 01/09976-3). 2 Pesquisador do Instituto Agronômico- IAC/APTA, Cx. Postal 28, 13012-970, Campinas-SP, teixeira@iac.sp.gov.br

${ }^{3}$ Professor Adjunto do Depto. de Solos e Adubos-FCAV/Unesp. Bolsista do CNPq. Jaboticabal-SP, natale @ fcav.unesp.br

${ }^{4}$ Pesquisador da APTA Regional Centro Norte. Pindorama-SP, lmartins@aptaregional.sp.gov.br
} 
efetiva no aumento de produtividade e, conseqüentemente, no lucro obtido pelos produtores.

Na Austrália, a fertirrigação em bananeira aumentou a eficiência do uso de fertilizantes, possibilitando reduzir as doses recomendadas para aplicação convencional entre 20 e $30 \%$ (Stewart et al., 1998). As principais vantagens da fertirrigação para as condições de cultivo australianas decorrem de que a aplicação regular de fertilizantes implica que a disponibilidade dos nutrientes se mantenha mais constante ao longo do ciclo, e eventuais precipitações de alta intensidade, causadoras de erosão ou lixiviação, tenham menor impacto sobre o crescimento das plantas. Esses autores também consideram que os fertilizantes na água de irrigação são aplicados nos locais de maior absorção e prontamente acessíveis às plantas e que perdas gasosas ou por escorrimento superficial são minimizadas com a fertirrigação. $\mathrm{Na}$ Índia, Srinivas (1997) obteve rendimentos de frutos semelhantes (em torno de $30 \mathrm{t} \mathrm{ha}^{1}$ ) com aplicação de $100 \mathrm{~g}$ de $\mathrm{N}$

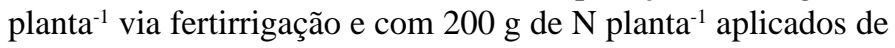
forma convencional. Hagin \& Tucker (1992) relataram maior aproveitamento do $\mathrm{N}$ por bananeiras com uréia aplicada por fertirrigação em relação à aplicação convencional na superfície do solo.

O objetivo deste trabalho foi determinar efeitos da aplicação de $\mathrm{N}$ e K em bananeira por meio de adubo sólido na superfície do solo e via fertirrigação.

\section{MATERIAL E MÉTODOS}

O experimento de campo foi estabelecido em dezembro de 2002, empregando-se delineamento experimental de blocos casualizados, com seis repetições. Os tratamentos (Tabela 1) constaram de frações da recomendação de adubação convencional de nitrogênio e potássio aplicada via fertirrigação e por meio de adubo sólido na superfície do solo. As doses de nitrogênio (350 kg de $\mathrm{N} \mathrm{ha}^{1}$ ano $^{1}$ ) e de potássio (400 $\mathrm{kg}$ de $\mathrm{K}_{2} \mathrm{O}$ ha ${ }^{1}$ ano ${ }^{1}$ ) foram calculadas seguindo-se as recomendações do Boletim 100 do Instituto Agronômico (Teixeira et al., 1997), as quais são baseadas na produtividade esperada e em atributos químicos do solo. Utilizou-se a variedade Nanicão (Grupo AAA, subgrupo Cavendish) no espaçamento de 2 × 2,5 m (2000 plantas ha ${ }^{1}$ ). $\mathrm{O}$ manejo do bananal seguiu as recomendações técnicas para a região, destacando-se que o controle de sigatoka foi realizado de forma preventiva, com aplicações mensais de fungicidas no período de outubro a março. Outros detalhes do experimento foram apresentados por Teixeira et al. (2006).

No primeiro ciclo de cultivo, fizeram-se medidas periódicas (aproximadamente a cada 30 dias) do comprimento do pseudocaule (solo até o topo da roseta foliar) e diâmetro a $30 \mathrm{~cm}$ do solo, na planta-mãe. Ajustou-se a seguinte função matemática (logística) para modelar o crescimento em função do tempo e tratamentos aplicados:

$$
Y=\frac{a}{1+\operatorname{EXP}(b-k X)} \text {, onde: }
$$

$X=$ tempo (dias);

$Y$ e $a=$ estimam a altura das plantas num dado tempo $(X)$ e altura máxima, respectivamente;

$\mathrm{b}$ e $\mathrm{k}=$ constantes.

$\mathrm{Na}$ época da emissão da inflorescência, foram medidos o diâmetro e o comprimento do pseudocaule e contadas as folhas ativas (com mais da metade do limbo verde). Na colheita do cacho, contaram-se novamente as folhas ativas. Calculou-se o índice de durabilidade das folhas, dado pela relação:

$$
I D F=100 \times \frac{N F C}{N F E} \quad \text {, na qual: }
$$

$I D F=$ índice de durabilidade foliar (\%);

$N F C$ = número de folhas ativas na época da colheita do cacho; $N F E=$ número de folhas ativas na época da emissão da inflorescência.

A taxa de crescimento absoluto do comprimento do pseudocaule foi estimada pela relação:

$$
T C A=\frac{C_{2}-C_{1}}{t_{2}-t_{1}}, \text { na qual: }
$$

$T C A=$ taxa de crescimento absoluto para o intervalo de tempo entre as medidas um e dois $\left(\mathrm{cm} \mathrm{dia}^{-1}\right) ; C_{1}=$ medida do comprimento do pseudocaule na época um $\left(t_{1}\right.$, em dias); $C_{2}=$ medida do comprimento do pseudocaule na época dois $\left(t_{2}\right.$, em dias). Para o primeiro ciclo de produção, $t_{1}$ coincide com o plantio e $t_{2}$, com a emissão da inflorescência; no segundo ciclo, $\mathrm{t}_{1}$ foi a época da emissão da inflorescência no ciclo anterior e $t_{2}$, coincidiu com a emissão no segundo ciclo.

O estado nutricional das plantas foi avaliado por meio de análise química da terceira folha, contada a partir do ápice, amostrada na época da emissão floral (Martin-Prével, 1984). As amostras foram processadas e analisadas quanto aos teores de $\mathrm{N}, \mathrm{P}, \mathrm{K}, \mathrm{Ca}, \mathrm{Mg}, \mathrm{B}, \mathrm{Cu}, \mathrm{Fe}, \mathrm{Mn}$ e Zn, de acordo com Bataglia et al. (1983). As relações K:N e K:Mg foram calculadas dividindo-se a concentração de $\mathrm{K}$ foliar pela concentração de $\mathrm{N}$ e $\mathrm{Mg}$, respectivamente.

Os cachos foram colhidos e pesados quando os frutos da penca dois ainda apresentavam quinas, correspondendo ao grau de maturação "3/4 magro" (Moreira, 1999), fazendo-se o despencamento e a contagem das pencas comercializáveis e frutos. Retirou-se uma amostra ao acaso de quatro frutos por cacho, nos quais se mediram comprimento (face convexa) e diâmetro maior. Uma das maneiras de avaliar a qualidade dos frutos, especialmente visando à sua comercialização, é medir comprimento e diâmetro, pois sua classificação baseia-se nestas dimensões (PBMH \& PIF, 2006).

Os dados foram analisados empregando-se o módulo GLM do Statistical Analysis System (SAS). Para testar a significância dos efeitos dos tratamentos, empregou-se o teste F; quando foram detectados efeitos significativos, os tratamentos foram comparados por meio do teste $t$ de Student $(\alpha=0,05)$. Para os tratamentos de doses de fertilizante aplicado via fertirrigação, foram ajustadas equações de regressão. 


\section{RESULTADOS E DISCUSSÃO}

O crescimento das plantas em função do tempo, no primeiro ciclo de cultivo, foi modelado ajustando-se funções logísticas [ $Y=a / l+\exp (b-k X)]$ para cada tratamento (Tabela 2). A partir dos intervalos de confiança (IC 95\%), calculados para o parâmetro $a$ (altura máxima), observou-se que os tratamentos não diferiram entre si; entretanto, a época de crescimento máximo foi antecipada pela adubação.

Calculou-se a taxa de crescimento absoluto do pseudocaule (TCA), a qual reflete o vigor da planta. Os tratamentos tiveram efeito significativo na TCA (Tabela 3). Na Figura 1, são apresentadas regressões entre as doses de $\mathrm{N}$ e $\mathrm{K}$ aplicadas via fertirrigação e a TCA, indicando que a adubação determinou plantas mais vigorosas. Observou-se, também, que a dose em torno de $80 \%$ da recomendação aplicada via fertirrigação determinou TCAs semelhantes às obtidas com a aplicação de $100 \%$ da dose de $\mathrm{N}$ e K.

Nos dois ciclos de produção, houve tendência de diminuição do ciclo em resposta à adubação (Tabela 3). A duração do primeiro ciclo variou inversamente à dose de adubo aplicado via fertirrigação (Figura 2). Nas condições de cultivo da região, ocorre atraso no desenvolvimento das plantas com aporte insuficiente de nitrogênio. Normalmente, o aporte de $\mathrm{N}$ regula essa variação, ocorrendo encurtamento na duração do ciclo proporcional ao fornecimento de $\mathrm{N}$, como descrito em Teixeira et al. (2002). Entretanto, os efeitos do N sobre a duração do ciclo relatados na literatura são divergentes: Lahav (1995) afirmou que incrementos no fornecimento de $\mathrm{N}$ causaram alongamento no ciclo, especialmente entre a emissão da inflorescência e colheita. Borges et al. (1997) observaram que doses crescentes de $\mathrm{N}$ prolongaram o ciclo em 'Prata-Anã'.

$\mathrm{Na}$ época da emissão da inflorescência, foram medidos comprimento e diâmetro do pseudocaule da planta-mãe (Tabela 3). O efeito positivo da adubação com $\mathrm{N}$ e $\mathrm{K}$ aplicada via fertirrigação no diâmetro das plantas é apresentado na Figura 3. O diâmetro do pseudocaule é uma variável importante, pois normalmente apresenta boa correlação com a produção das plantas (Soto, 1992).

Observou-se que foi possível manter boa parte das folhas vivas durante o enchimento dos cachos nos dois ciclos de cultivo, com índice de durabilidade foliar (IDF) $>70 \%$ em todos os tratamentos (Tabela 3), independentemente das doses de $\mathrm{N}$ e $\mathrm{K}$, e forma de aplicação dos fertilizantes. A manutenção de área foliar ativa por mais tempo é importante para a produção de frutos em bananeira, o que normalmente justifica o dispendioso controle de doenças foliares (Sigatoka, principalmente). Em relação à fertilidade do solo, há relatos de que o suprimento adequado de K seja fundamental para a preservação da área foliar (Hasselo, 1961; Lahav, 1972; Lahav,1995). A senescência das folhas em bananeiras, entre a época da emissão da inflorescência e a colheita dos cachos, também pode ser acelerada em condições de desequilíbrio nutricional de $\mathrm{N}$ e K (Teixeira et al., 2001).

Os efeitos dos tratamento sobre os teores foliares de N, $\mathrm{K}, \mathrm{P}, \mathrm{Ca}$ e Mg, nos dois ciclos de cultivo, são apresentados na Tabela 4. Observou-se que os tratamentos tiveram efeito significativo, principalmente nos teores foliares de N e K.

Segundo Lahav (1995), o nível crítico (NC) para o teor foliar de $\mathrm{N}$ é $26 \mathrm{~g} \mathrm{~kg}^{1}$. No primeiro ciclo de cultivo, com exceção do tratamento sem adubação nitrogenada, os demais forneceram nitrogênio suficiente para atingir o NC. Neste ciclo, a variação no teor foliar de $\mathrm{N}$ foi proporcional à quantidade de nitrogênio aplicado via fertirrigação (Figura 4). De acordo com a regressão apresentada na Figura 4, para alcançar o teor foliar de $\mathrm{N}$ obtido com a aplicação de $350 \mathrm{~kg} \mathrm{ha}^{1}$ de $\mathrm{N}$ via solo (29,2 $\left.\mathrm{g} \mathrm{kg}^{1}\right)$, estimase que seriam necessários cerca de $312 \mathrm{~kg}$ ha ${ }^{1}$ de $\mathrm{N}$ aplicados por fertirrigação. $\mathrm{O}$ teor foliar de $\mathrm{N}$, próximo ao $\mathrm{NC}$, observado nas plantas sem adubação, indica que a mineralização de matéria orgânica do solo, provavelmente, supriu boa parte da necessidade de N. Para o segundo ciclo, não foi possível ajustar função matemática para modelar o efeito dose de $\mathrm{N}$ via fertirrigação no seu teor foliar. Estes resultados podem ter sido afetados por efeito diluição, visto que não houve diferença entre o tratamento sem $\mathrm{N}$ e aquele com aplicação de 1,2 vez a dose de $\mathrm{N}$ recomendada (Tabela 4), mesmo com teores de N abaixo do NC. Neste ciclo, a aplicação de $\mathrm{N}$ via solo apresentou tendência de ser mais eficiente do que por fertirrigação, pelo menos em relação ao seu efeito no teor foliar de N.

Para potássio, o efeito dos tratamentos não pode ser modelado por função matemática. Como as doses de $\mathrm{K}$ aumentaram concomitantemente às de $\mathrm{N}$ que, por sua vez, tem efeito positivo no crescimento das plantas, o efeito diluição manifestou-se fortemente. Nos dois ciclos de cultivo, na área irrigada e sem aplicação de K e N, obtiveram-se teores foliares de K que não diferiram daqueles da maior dose via fertirrigação ou via solo (Tabela 4). O NC para potássio foliar - $30 \mathrm{~g} \mathrm{~kg}^{1}$, segundo Lahav (1995) - não foi alcançado nem com a maior dose de adubo. Em trabalhos desenvolvidos nas condições de cultivo do Estado de São Paulo, observou-se que o NC para potássio não foi atingido mesmo com adubação adequada e com plantas apresentando produtividades elevadas (Teixeira et al., 2002; Damatto Jr., 2005).

Nos dois ciclos de cultivo, os teores foliares de $\mathrm{Ca}$ e $\mathrm{Mg}$ (Tabela 4) foram sempre superiores aos NC, 5,0 e 3,0 $\mathrm{g} \mathrm{kg}^{-1}$, respectivamente, apresentados por Lahav (1995). Também não foi observado efeito negativo do fornecimento de $\mathrm{K}$ sobre os teores de $\mathrm{Ca}$ e $\mathrm{Mg}$.

A relação K:N na folha-índice (Tabela 4) ficou aquém de 1,4, valor que, segundo Teixeira et al. (2001), determinaria maior durabilidade foliar devido ao equilíbrio nutricional em relação a estes nutrientes. Entretanto, observou-se que a durabilidade foliar não foi afetada, pois o IDF foi sempre superior a $70 \%$, independentemente dos tratamentos (Tabela 3). A relação entre as concentrações foliares de $\mathrm{K}$ e $\mathrm{Mg}$ pode indicar a ocorrência de um desequilíbrio conhecido como "azul-da-bananeira". Teores de $\mathrm{K}: \mathrm{Mg}\left(\mathrm{g} \mathrm{kg}^{-1}\right)$ maiores que 14,7 foram associados ao "azul-dabananeira" em Santa Catarina por Lichtemberg \& Malburg (1983). Os valores de K:Mg apresentados na Tabela 4 estão abaixo do limite crítico para o "azul-da-bananeira" e também são inferiores à faixa ideal $(8,1$ a 11,4), sugerida por Borges \& Oliveira (2000).

Nos dois ciclos de cultivo, a produção de frutos variou em função dos tratamentos (Tabela 5). No primeiro ciclo, a 

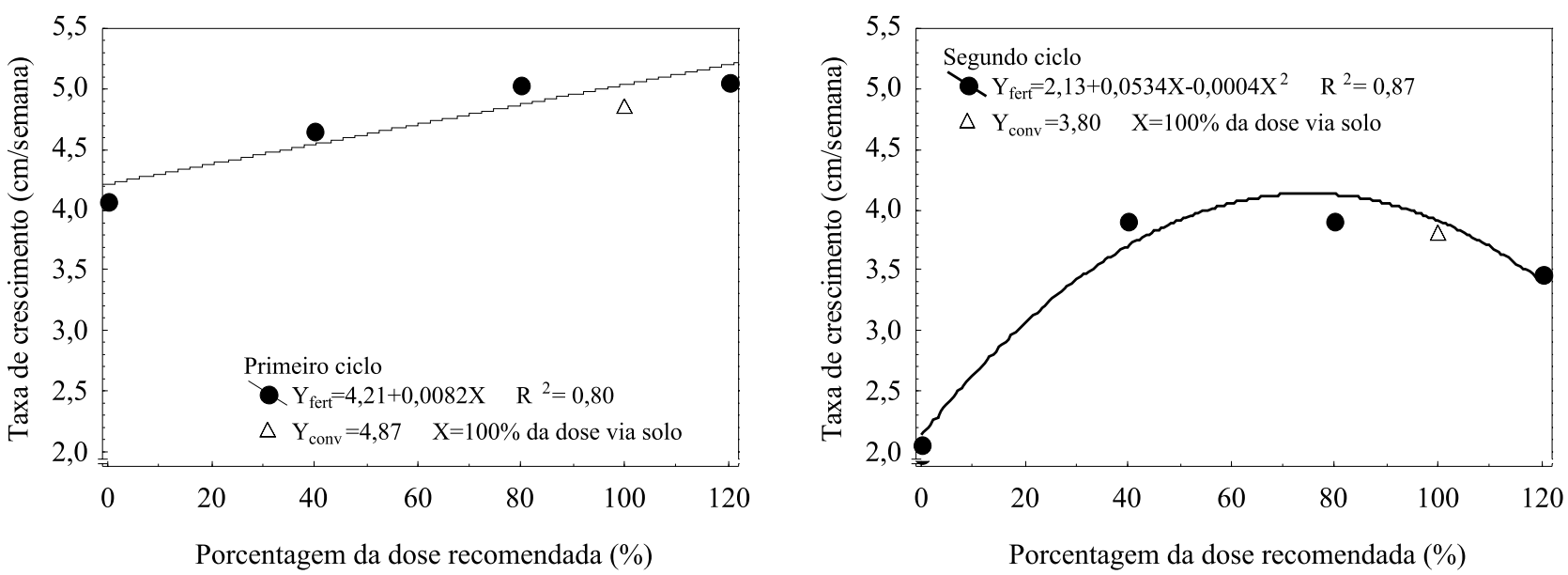

FIGURA 1 - Taxa de crescimento de bananeira em função de fertirrigação e adubação convencional com N e K, no primeiro (esq.) e segundo (dir.) ciclos de produção.



FIGURA 2 - Duração do primeiro ciclo de produção de bananeira em função de fertirrigação e adubação convencional com $\mathrm{N}$ e $\mathrm{K}$.

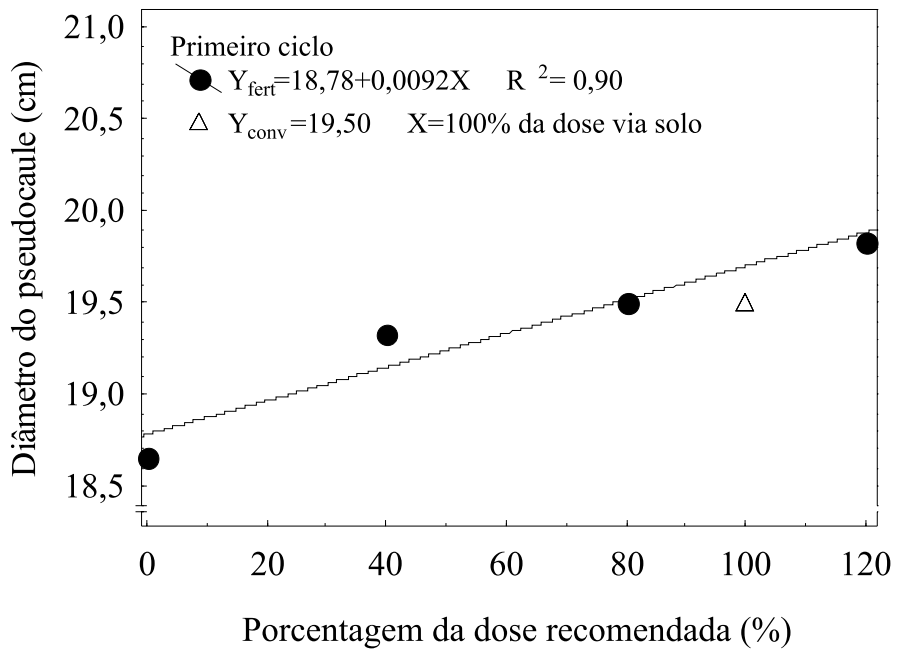

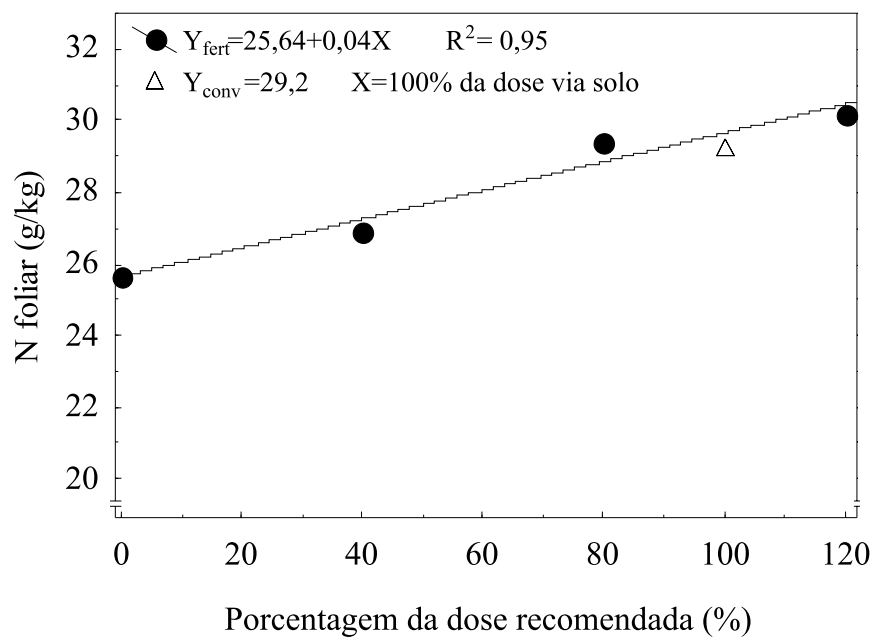

FIGURA 4 - Teor de $\mathrm{N}$ foliar de bananeira em função de fertirrigação e adubação convencional com $\mathrm{N}$ e K.

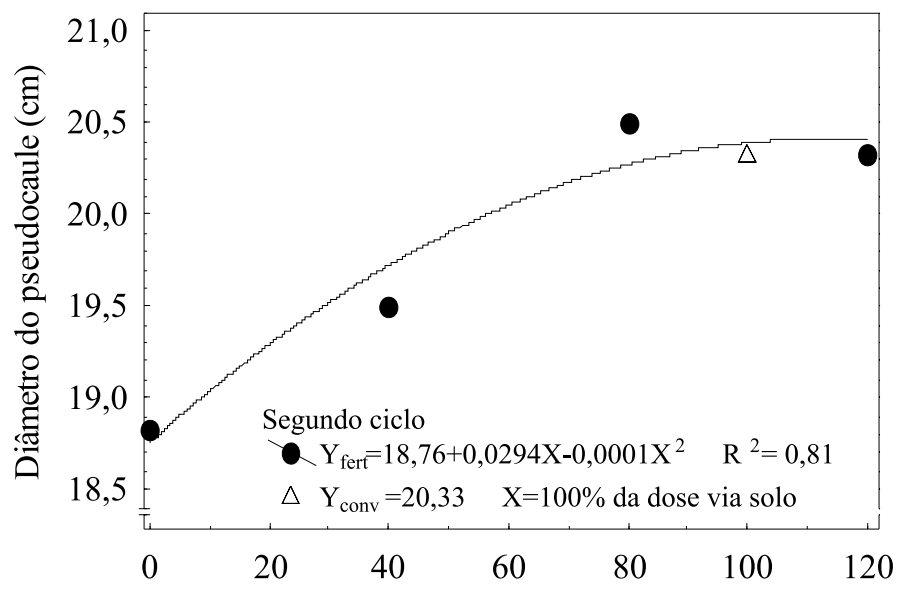

Porcentagem da dose recomendada (\%)

FIGURA 3 - Diâmetro do pseudocaule de bananeira em função de fertirrigação e adubação convencional com N e K, no primeiro (esq.) e segundo (dir.) ciclos de produção. 


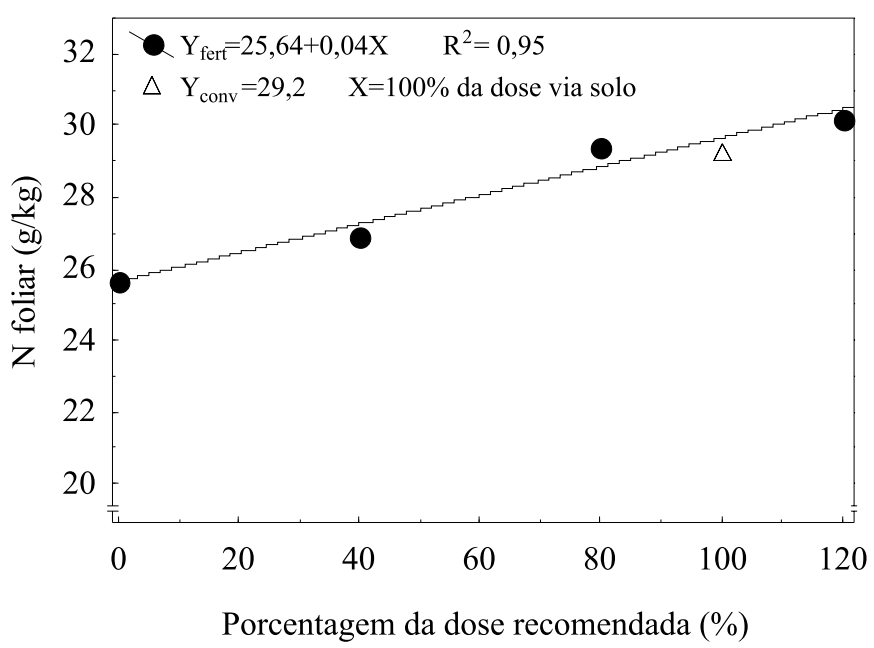

FIGURA 4 - Teor de $\mathrm{N}$ foliar de bananeira em função de fertirrigação e adubação convencional com $\mathrm{N}$ e K.

produção diminuiu em resposta ao aumento das doses de adubo aplicado via fertirrigação. Este comportamento, provavelmente, deveu-se ao encurtamento do período plantio-emisão em resposta ao aumento no fornecimento de N. É possível que o aporte crescente de $\mathrm{N}$, ao causar redução significativa na duração do ciclo, tenha determinado que plantas ainda com reservas insuficientes de nutrientes começassem a produzir. Este comportamento é semelhante ao relatado por Teixeira et al. (2002) para bananeiras cultivadas no Planalto Paulista.

Ao final da segunda safra, quando a produção de frutos acumulada nos dois ciclos foi expressa em tha ${ }^{1}$ ano ${ }^{1}$ (que é o que interessa para o produtor, visto que os custos de produção são proporcionais à área cultivada e à duração do ciclo de produção), o incremento na dose de adubo aplicado por fertirrigação apresentou efeito positivo (Tabela 5). Com a aplicação de aproximadamente $80 \%$ da dose recomendada, foi possível atingir produção equivalente a $100 \%$ da dose via adubo sólido (Figura 5). A possibilidade de reduzir a quantidade de adubo aplicado via fertirrigação, em comparação com a adubação convencional, foi citada por vários autores, como Srinivas (1997), na Índia, Stewart et al. (1998), na Austrália e Hagin \& Tucker (1992), todos creditando essa redução ao possível maior aproveitamento dos fertilizantes quando aplicados junto com a irrigação.

A quantidade de pencas e de frutos por cacho responderam positivamente à adubação no segundo ciclo de produção (Tabela 5). Na Tabela 5, observa-se que os tratamentos não influenciaram significativamente na dimensão dos frutos colhidos. Independentemente da adubação, os frutos apresentaram comprimento superior a $22 \mathrm{~cm}$ e diâmetro maior que $32 \mathrm{~mm}$, valores mínimos para classificá-los como "extra", segundo as normas do Programa Brasileiro para Modernização da Horticultura e Produção Integrada de Frutas (PBMH \& PIF, 2006).

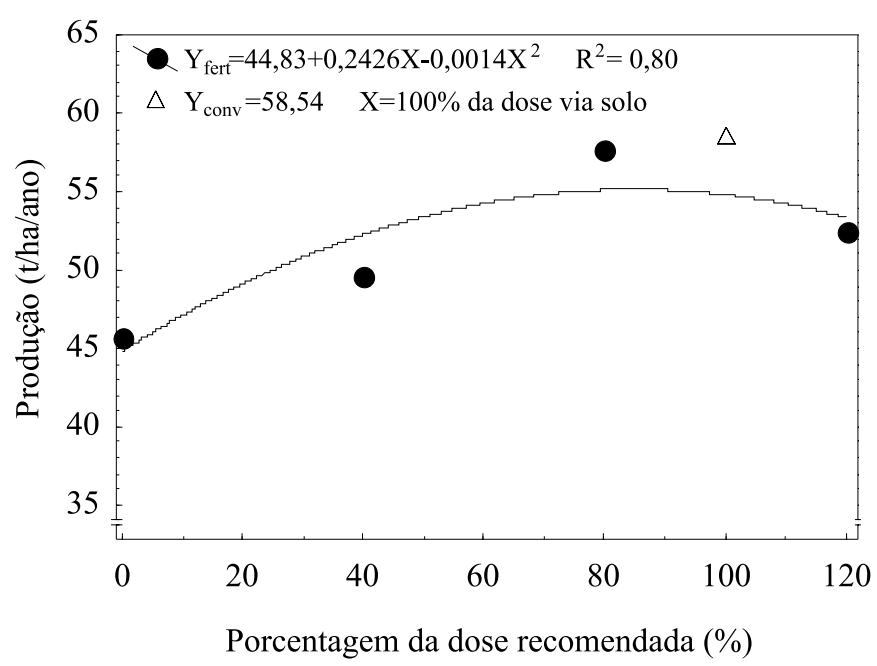

FIGURA 5 - Produção de frutos em função de fertirrigação e adubação convencional com $\mathrm{N}$ e K em bananeira.

TABELA 1 - Tratamentos aplicados às unidades experimentais

\begin{tabular}{cl}
\hline Tratamento & \multicolumn{1}{c}{ Descrição } \\
\hline T1 & Irrigação sem aplicação de $\mathrm{Ne} \mathrm{K}$ \\
T2 & Fertirrigação com $40 \%$ da dose de $\mathrm{N} \mathrm{e} \mathrm{K}{ }^{(1)}$ \\
T3 & Fertirrigação com $80 \%$ da dose de $\mathrm{N} \mathrm{e} \mathrm{K}^{(1)}$ \\
$\mathrm{T} 4$ & Fertirrigação com $120 \%$ da dose de $\mathrm{N} \mathrm{e} \mathrm{K}^{(1)}$ \\
T5 & Irrigação com $100 \%$ da dose de $\mathrm{N} \mathrm{e} \mathrm{K}{ }^{(1)}$ via solo \\
\hline
\end{tabular}

(1) Dose de N: $350 \mathrm{~kg}$ de $\mathrm{N} \mathrm{ha}^{1}$ ano $^{1}$; dose de $\mathrm{K}: 400 \mathrm{~kg}$ de $\mathrm{K}_{2} \mathrm{O}$ ha ${ }^{1} \mathrm{ano}^{1}$.

TABELA 2 - Parâmetros da função logística [ $Y=a / l+\exp (b-k X)]$ que relaciona a altura das bananeiras $(\mathrm{Y}, \mathrm{cm}) \mathrm{em}$ função do tempo (X, dias), sob fertirrigação e adubação convencional.

\begin{tabular}{|c|c|c|c|c|}
\hline Tratamento & & Parâmetro & $\mathbf{R}^{2}$ & $\begin{array}{c}\text { Crescimento } \\
\text { máximo }^{(1)}\end{array}$ \\
\hline \multirow{4}{*}{$\begin{array}{l}\text { T1-Irriga } \\
\text { dose } 0\end{array}$} & & & \multirow{4}{*}{0,92} & --- dias --- \\
\hline & $a$ & $201,43 \pm 12,62$ & & \multirow{3}{*}{151} \\
\hline & $b$ & $2,18 \pm 0,29$ & & \\
\hline & $k$ & $0,0144 \pm 0,002$ & & \\
\hline \multirow{3}{*}{$\begin{array}{c}\text { T2-Fertirriga } \\
\text { dose } 40 \%\end{array}$} & $a$ & $207,54 \pm 9,25$ & \multirow{3}{*}{0,95} & \multirow{3}{*}{141} \\
\hline & $b$ & $2,35 \pm 0,26$ & & \\
\hline & $k$ & $0,0167 \pm 0,002$ & & \\
\hline \multirow{3}{*}{$\begin{array}{c}\text { T3-Fertirriga } \\
\text { dose } 80 \%\end{array}$} & $a$ & $187,82 \pm 7,90$ & \multirow{3}{*}{0,92} & \multirow{3}{*}{115} \\
\hline & $b$ & $2,91 \pm 0,50$ & & \\
\hline & $k$ & $0,0254 \pm 0,005$ & & \\
\hline \multirow{3}{*}{$\begin{array}{c}\text { T4-Fertirriga } \\
\text { dose } 120 \%\end{array}$} & $a$ & $204,41 \pm 5,49$ & \multirow{3}{*}{0,97} & \multirow{3}{*}{124} \\
\hline & $b$ & $2,67 \pm 0,24$ & & \\
\hline & $k$ & $0,0216 \pm 0,002$ & & \\
\hline T5-Irriga & $a$ & $204,12 \pm 7,69$ & \multirow{3}{*}{0,96} & \multirow{3}{*}{135} \\
\hline dose $100 \%$ & $b$ & $2,73 \pm 0,29$ & & \\
\hline via solo & $k$ & $0,0202 \pm 0,002$ & & \\
\hline
\end{tabular}

Rev. Bras. Frutic., Jaboticabal - SP, v. 29, n. 1, p. 153-160, Abril 2007 
TABELA 3 - Comprimento e diâmetro do pseudocaule, taxa de crescimento absoluto, duração do ciclo, número de folhas e índice de durabilidade foliar em função de fertirrigação e adubação convencional com N e K, em dois ciclos de cultivo de bananeira.

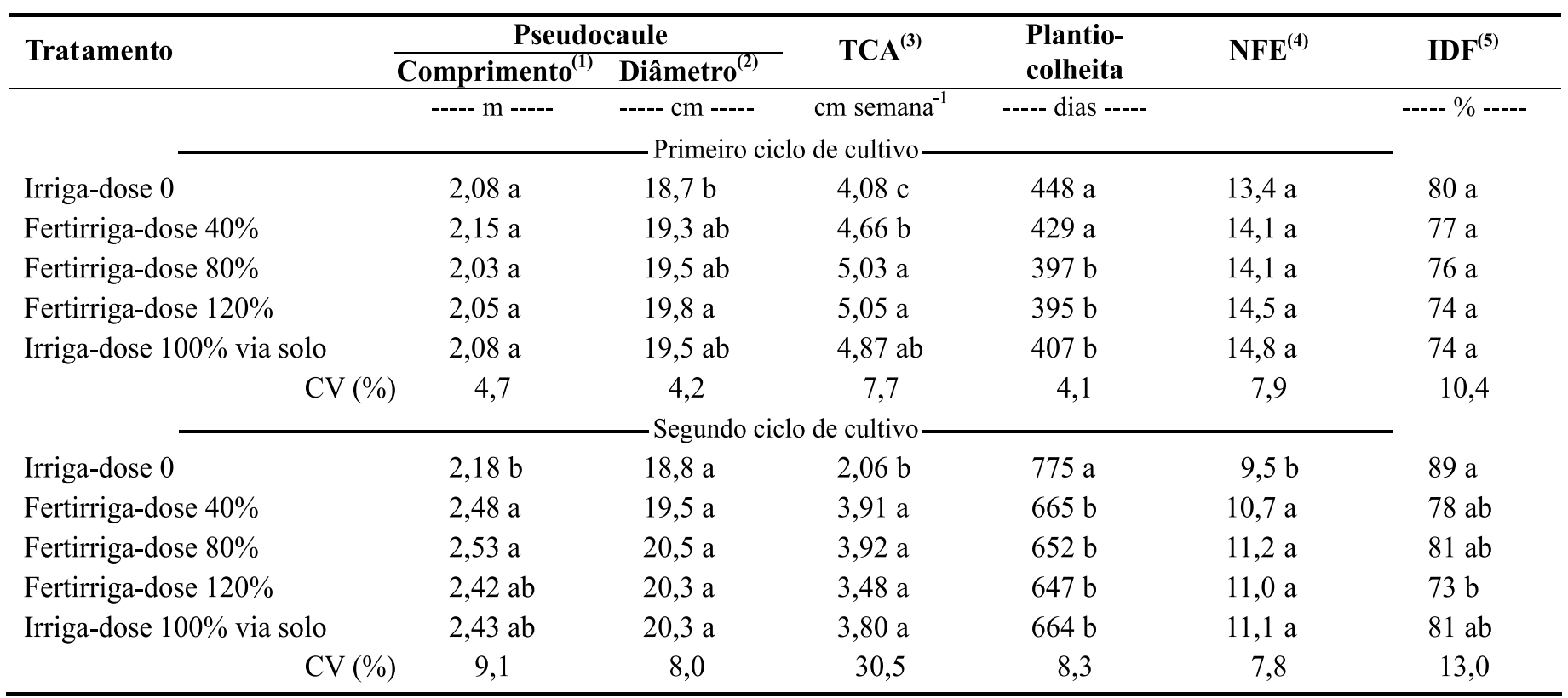

Valores de um mesmo ciclo de cultivo seguidos por letras iguais não diferem entre si, pelo teste t de Student $(p>0,05)$. ${ }^{(1)}$ Medido do solo à roseta foliar e ${ }^{(2)}$ medido a $30 \mathrm{~cm}$ do solo na época da emissão floral; ${ }^{(3)}$ taxa de crescimento absoluto do pseudocaule estimada entre o plantio e a emissão floral do primeiro ciclo e da emissão floral da planta-mãe até a emissão no segundo ciclo; ${ }^{(4)}$ número de folhas contadas na época da emissão foliar; ${ }^{(5)}$ IDF=(Número de folhas na colheita $\div$ número na emissão) $\times 100$.

TABELA 4 - Teores de nutrientes e relações K:N e K:Mg na folha-índice de bananeira em função de fertirrigação e adubação convencional com $\mathrm{N}$ e $\mathrm{K}$, em dois ciclos de cultivo.

\begin{tabular}{|c|c|c|c|c|c|c|c|c|}
\hline Tratamento & & $\mathbf{N}$ & $\mathbf{K}$ & $\mathbf{P}$ & $\mathbf{C a}$ & Mg & $\mathbf{K}: \mathbf{N}$ & K:Mg \\
\hline \multicolumn{9}{|c|}{ 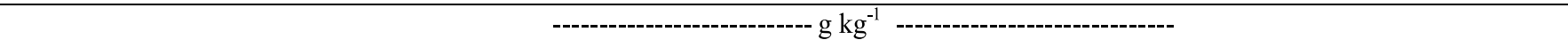 } \\
\hline Irriga-dose 0 & & $25,7 \mathrm{~b}$ & $27,0 \mathrm{ab}$ & $1,9 \mathrm{ab}$ & $12,0 \mathrm{~b}$ & $4,8 \mathrm{c}$ & $1,05 \mathrm{a}$ & $5,65 \mathrm{a}$ \\
\hline Fertirriga-dose $40 \%$ & & $26,9 \mathrm{~b}$ & $25,0 \mathrm{c}$ & $1,7 \mathrm{~b}$ & $13,8 \mathrm{~b}$ & $5,4 \mathrm{abc}$ & $0,93 \mathrm{bc}$ & $4,75 \mathrm{ab}$ \\
\hline Fertirriga-dose $80 \%$ & & 29,4 a & $26,5 \mathrm{bc}$ & $2,0 \mathrm{a}$ & $17,5 \mathrm{a}$ & $6,0 \mathrm{ab}$ & $0,90 \mathrm{bc}$ & $4,61 \mathrm{ab}$ \\
\hline Fertirriga-dose $120 \%$ & & $30,2 \mathrm{a}$ & $25,5 \mathrm{bc}$ & $1,9 \mathrm{ab}$ & $15,6 \mathrm{ab}$ & $6,1 \mathrm{a}$ & $0,87 \mathrm{c}$ & $4,33 \mathrm{~b}$ \\
\hline \multirow[t]{2}{*}{ Irriga-dose $100 \%$ via solo } & & $29,2 \mathrm{~b}$ & $28,1 \mathrm{a}$ & $1,7 \mathrm{~b}$ & $11,9 \mathrm{~b}$ & $5,1 \mathrm{bc}$ & $0,96 \mathrm{~b}$ & $5,66 \mathrm{a}$ \\
\hline & $\mathrm{CV}(\%)$ & 4,2 & 4,8 & 11,9 & 21,0 & 16,2 & 7,4 & 19,5 \\
\hline & & & - Segundo & o de cult & & & & \\
\hline Irriga-dose 0 & & $18,6 \mathrm{bc}$ & $26,8 \mathrm{a}$ & $2,4 \mathrm{a}$ & $10,0 \mathrm{~b}$ & $3,1 \mathrm{~b}$ & $1,47 \mathrm{a}$ & $8,83 \mathrm{a}$ \\
\hline Fertirriga-dose $40 \%$ & & $17,8 \mathrm{c}$ & $22,7 \mathrm{ab}$ & $1,4 \mathrm{~b}$ & $14,8 \mathrm{a}$ & $4,1 \mathrm{a}$ & $1,28 \mathrm{ab}$ & $5,64 \mathrm{~b}$ \\
\hline Fertirriga-dose $80 \%$ & & $20,3 \mathrm{ab}$ & $23,3 \mathrm{ab}$ & $1,2 \mathrm{~b}$ & $15,0 \mathrm{a}$ & $4,3 \mathrm{a}$ & $1,15 \mathrm{bc}$ & $5,67 \mathrm{~b}$ \\
\hline Fertirriga-dose $120 \%$ & & $20,3 \mathrm{ab}$ & $21,7 \mathrm{~b}$ & $1,3 \mathrm{~b}$ & $17,4 \mathrm{a}$ & $4,6 \mathrm{a}$ & $1,08 \mathrm{bc}$ & $4,71 \mathrm{~b}$ \\
\hline \multirow[t]{2}{*}{ Irriga-dose $100 \%$ via solo } & & $21,8 \mathrm{a}$ & $21,3 \mathrm{~b}$ & $1,2 \mathrm{~b}$ & $17,2 \mathrm{a}$ & $4,6 \mathrm{a}$ & $0,98 \mathrm{c}$ & $4,87 \mathrm{~b}$ \\
\hline & $\mathrm{CV}(\%)$ & 9,8 & 18,2 & 25,9 & 17,6 & 14,7 & 18,6 & 23,7 \\
\hline
\end{tabular}

Valores de um mesmo ciclo de cultivo seguidos por letras iguais não diferem entre si, pelo teste t de Student $(p>0,05)$. 
TABELA 5 - Produção de frutos em função de fertirrigação e adubação convencional com N e K, em dois ciclos de cultivo de bananeira.

\begin{tabular}{|c|c|c|c|c|c|c|c|}
\hline \multirow[t]{2}{*}{ Tratamento } & \multirow{2}{*}{$\begin{array}{c}\text { Cacho } \\
---\mathrm{kg}---\end{array}$} & \multicolumn{2}{|c|}{ Produção } & \multicolumn{3}{|c|}{ Pencas/cacho Frutos/cacho Comprimento } & \multirow{2}{*}{$\begin{array}{l}\text { Diâmetro } \\
---\mathrm{mm} \text {--- }\end{array}$} \\
\hline & & $---\mathrm{t} \mathrm{ha}^{-1}---$ & $\mathrm{tha}^{-1}$ ano $^{-1(1)}$ & & & $---\mathrm{cm} \mathrm{---}$ & \\
\hline Irriga-dose 0 & $27,7 \mathrm{a}$ & $55,3 \mathrm{a}$ & $45,2 \mathrm{a}$ & $8,5 \mathrm{a}$ & $124 \mathrm{a}$ & $22,8 \mathrm{a}$ & $40 \mathrm{a}$ \\
\hline Fertirriga-dose $40 \%$ & $26,2 \mathrm{ab}$ & $52,3 \mathrm{ab}$ & $44,7 \mathrm{a}$ & $7,9 \mathrm{a}$ & $123 \mathrm{a}$ & $22,5 \mathrm{a}$ & $38 \mathrm{~b}$ \\
\hline Fertirriga-dose $80 \%$ & $26,3 \mathrm{ab}$ & $52,7 \mathrm{ab}$ & $48,4 \mathrm{a}$ & $8,4 \mathrm{a}$ & $128 \mathrm{a}$ & $22,8 \mathrm{a}$ & $39 \mathrm{ab}$ \\
\hline Fertirriga-dose $120 \%$ & $23,4 \mathrm{~b}$ & $46,8 \mathrm{~b}$ & 43,4 a & $8,3 \mathrm{a}$ & $129 \mathrm{a}$ & $22,1 \mathrm{a}$ & $38 \mathrm{~b}$ \\
\hline Irriga-dose $100 \%$ via solo & $26,0 \mathrm{ab}$ & $52,0 \mathrm{ab}$ & $46,9 \mathrm{a}$ & $8,6 \mathrm{a}$ & $132 \mathrm{a}$ & $22,6 \mathrm{a}$ & $39 \mathrm{ab}$ \\
\hline $\mathrm{CV}(\%)$ & 11,0 & 11,0 & 11,4 & 7,7 & 9,5 & 6,3 & 3,7 \\
\hline Irriga-dose 0 & $20,5 \mathrm{ab}$ & $\overline{41,0 \mathrm{ab}} \mathrm{Se}$ & $\begin{array}{c}\text { Indo ciclo de c } \\
45,7 \mathrm{~b}\end{array}$ & altivo $\overline{7,7 \mathrm{~b}}$ & $106 \mathrm{c}$ & $21,9 \mathrm{a}$ & $39 \mathrm{a}$ \\
\hline Fertirriga-dose $40 \%$ & $19,1 \mathrm{~b}$ & $38,1 \mathrm{~b}$ & $49,6 \mathrm{ab}$ & $7,8 \mathrm{ab}$ & $120 \mathrm{bc}$ & $23,5 \mathrm{a}$ & $38 \mathrm{a}$ \\
\hline Fertirriga-dose $80 \%$ & $24,9 \mathrm{ab}$ & $49,8 \mathrm{ab}$ & $57,7 \mathrm{a}$ & $8,9 \mathrm{a}$ & $147 \mathrm{a}$ & $22,5 \mathrm{a}$ & $43 \mathrm{a}$ \\
\hline Fertirriga-dose $120 \%$ & $23,1 \mathrm{ab}$ & $46,2 \mathrm{ab}$ & $52,5 \mathrm{ab}$ & $8,3 \mathrm{ab}$ & $127 \mathrm{abc}$ & $23,7 \mathrm{a}$ & $38 \mathrm{a}$ \\
\hline Irriga-dose $100 \%$ via solo & $26,8 \mathrm{a}$ & $53,6 \mathrm{a}$ & $58,5 \mathrm{a}$ & $8,8 \mathrm{a}$ & $139 \mathrm{ab}$ & $22,5 \mathrm{a}$ & $42 \mathrm{a}$ \\
\hline $\mathrm{CV}(\%)$ & 27,9 & 27,9 & 17,5 & 11,2 & 15,9 & 11,0 & 14,8 \\
\hline
\end{tabular}

Valores de um mesmo ciclo de cultivo seguidos por letras iguais não diferem entre si, pelo teste t de Student $(p>0,05)$. ${ }^{(1)}$ No segundo ciclo, trata-se de produção acumulada das duas safras: (produção safra1+produção safra2)/ano.

\section{CONCLUSÕES}

1. A adubação com $\mathrm{N}$ e K diminuiu o ciclo de produção.

2. A fertirrigação possibilitou reduzir a dose de $\mathrm{N}$ e K em relação à adubação convencional, sem prejuízo na produção de frutos.

\section{REFERÊNCIAS}

BAR-YOSEF, B. Advances in fertigation. In: Sparks, D.L. (Ed.). Advances in agronomy. New York: Academic Press, 1999. p.1-77.

BATAGLIA, O.C.; FURLANI, A.M.C.; TEIXEIRA, J.P.F.; FURLANI, P.R.; GALLO, J.R. Métodos de análise química de plantas. Campinas: IAC, 1983. 48p. (Boletim Técnico, 78).

BORGES, A.L.; OLIVEIRA, A.M.G. Nutrição, adubação e calagem. In: CORDEIRO, Z.J.M. (Ed.). Banana. produção: aspectos técnicos. Brasília: Embrapa, 2000. p. 47-59.

BORGES, A.L.; SILVA, J.T.A.; OLIVEIRA, S.L. Adubação nitrogenada e potássica para bananeira cv. Prata Anã irrigada: produção e qualidade dos frutos no primeiro ciclo. Revista Brasileira de Fruticultura, Cruz das Almas, v.19, p.179-84, 1997

DAMATTO JR., E.R. Efeitos da adubação com composto orgânico na fertilidade do solo, desenvolvimento, produção e qualidade de frutos de bananeira 'Prata-anã' (Musa AAB). 2005. 70f. Dissertação (Mestrado em Agronomia) - Faculdade de Ciências Agronômicas, Universidade Estadual Paulista, Botucatu, 2005.

HAGIN, J.; TUCKER, B. Fertilization of dryland and irrigated soils. Berlin: Springer-Verlag, 1982. 190p.

HASSELO, H.N. Premature yellowing of Lacatan bananas. Tropical Agriculture, London, v.38, p.29-34, 1961.
HERNANDEZ, F.B.T. Potencialidades da fertirrigação. In: SIMPÓSIO BRASILEIRO SOBRE FERTILIZANTES FLUIDOS, 1994. Piracicaba. Anais... Piracicaba: ESALQ; CENA; POTAFOS, 1994. p. 215-25.

IBGE. Instituto Brasileiro de Geografia e Estatística. Disponível em: <http://www.ibge.gov.br>. Acesso em: 12 dez. 2006.

IEA. Instituto de Economia Agrícola do Estado de São Paulo. Disponível em: < http://www.iea.sp.gov.br >. Acesso em: 12 dez. 2006.

LAHAV E.; TURNER, D.W. Banana nutrition. Berna: IPI, 1983. 62p. (Bulletin, 7)

LAHAV, E. Banana nutrition. In: GOWEN, S. (Ed.). Bananas and plantains. London: Chapman \& Hall, 1995. p.258-316.

LAHAV, E. Effect of different amount of potassium on the growth of the banana. Tropical Agriculture, Guildford, v.49, p.321-35, 1972.

LICHTEMBERG, L.A.; MALBURG, J.L. Controle do azul-dabananeira pela aplicação de calcário dolomítico. Florianópolis: EMPASC, 1983.7p. (Comunicado Técnico, 67)

MARTIN-PRÉVEL, P. Bananier. In: MARTIN-PRÉVEL, P.; GAGNARD, J.; GAUTIER, P. (Ed.). L'analyse végétale dans le contrôle de l'alimentation des plantes tempérées et tropicales. Paris: Tec\&Doc, 1984. p.715-51.

MOREIRA, R.S. Banana: teoria e prática de cultivo. 2.ed. São Paulo: Fundação Cargill, 1999. CD-ROM

PBMH \& PIF - PROGRAMA BRASILEIRO PARA A MODERNIZAÇÃO DA HORTICULTURA E PRODUÇÃO INTEGRADA DE FRUTAS. Normas de classificação de banana. São Paulo: CEAGESP, 2006. 4p. (Documentos, 29)

SOTO, M. Bananos: cultivo y comercialización. $2^{\text {nd }}$ ed. San José: LIL, $1992.674 \mathrm{p}$.

SRINIVAS, K. Growth, yield, and quality of banana in relation to $\mathrm{N}$ fertigation. Tropical Agriculture, Trinidad, v.74, n.4, p.2604, 1997. 
STEWART, L.; CAMPAGNOLO, D.; DANIELLS, J.; LEMIN, C.; GOEBEL, R.; PINESE, B.; PETERSON, R.; PETERSON, R.; EVANAS, D.; PATTSON, T., ARMOUR, J., GUNTHER, $M$. Tropical banana information kit. Nambour: Queensland Department of Primary Industries, 1998. (Serie: Agrilink)

TEIXEIRA, L.A.J.; NATALE, W.; RUGGIERO, C. Alterações em alguns atributos químicos do solo decorrentes da irrigação e adubação nitrogenada e potássica em bananeira após dois ciclos de cultivo. Revista Brasileira de Fruticultura, Jaboticabal, v.23, p.684-689, 2001.

TEIXEIRA, L.A.J.; NATALE, W.; RUGGIERO, C. Nitrogen and potassium fertilization of 'Nanicão' banana (Musa AAA Cavendish subgroup) under irrigated and non-irrigated conditions. Acta Horticulturae, Leuven, v.275, p.771-9, 2002.

TEIXEIRA, L.A.J.; RUGGIERO, C.; NATALE, W. Manutenção de folhas ativas em bananeira- 'Nanicão' por meio do manejo das adubações nitrogenada e potássica e da irrigação. Revista Brasileira de Fruticultura, Jaboticabal, v.23, p.699703, 2001.

TEIXEIRA, L.A.J.; SPIRONELLO, A.; QUAGGIO, J.A.; FURLANI, P. Banana. In: RAIJ, B. van et al. (Ed.). Recomendações de adubação e calagem para o Estado de São Paulo. 2.ed.rev. Campinas: IAC, 1997. p.131-2. (Boletim Técnico, 100)

TEIXEIRA, L.A.J.; MARTINS, A.L.M.; NATALE, W.; BETTIOL NETO, J.E. Nitrogênio e potássio em bananeira via fertirrigação e adubação convencional-atributos químicos do solo. Revista Brasileira de Fruticultura, Jaboticabal, v.29, p.143-152, 2006.

VILLAS BÔAS, R.L.; FERNANDES, D.M.; BOARETTO, A.E., GODOY, L.G. Fertirrigação: uso e manejo. In: CONGRESSO BRASILEIRO DE CIÊNCIA DO SOLO, 30., 2005, Recife. Anais... SBCS, 2005.

YAGÜE, J.L.F. Fertirrigación. In:__ Técnicas de riego. $2^{\text {nd }}$ ed. Madri: MAPA/Mundi Prensa, 1996. p.343-61. 